\title{
Editorial
}

\section{Discovery of the ubiquitin proteasome system and its involvement in apoptosis}

\author{
G Melino ${ }^{1, *}$, on behalf of The Editors \\ ${ }^{1}$ Cell Death Differentiation Editorial Office, \\ www.nature.com/cdd \\ * Corresponding author: G Melino, Cell Death Differentiation Editorial Office, \\ www.nature.com/cdd; E-mail: cell.death.differ@uniroma2.it
}

\section{Cell Death and Differentiation (2005) 12, 1155-1157. doi:10.1038/sj.cdd.4401740}

This special issue of Cell Death and Differentiation has come to fruition thanks to the generous assistance of the Nobel Foundation in Stockholm, http://www.nobelprize.org ( ${ }^{(} \mathrm{T}$ The Nobel Foundation 2004). It is an honour and privilege to write a short introductory editorial to lectures ${ }^{1-3}$ and interviews ${ }^{4-6}$ with Aaron Ciechanover, Avram Hershko and Irwin Rose, the 2004 Nobel Laureates in Chemistry, for their pioneering work on the discovery of ubiquitin-mediated protein degradation. This is an additional tribute to these scientists, in recognition of their discovery and characterisation of the ubiquitin proteasome system (UPS), made in a small laboratory by great scientists with limited material, but not intellectual, resources. A detailed disquisition of the discovery of the UPS may be found in the very recent review published by Ciechanover. ${ }^{7} \mathrm{~A}$ brief, limited timeline covering the identification of the UPS is shown in Figure 1.

Hershko, Rose and Ciechanover, in their respective Nobel Lectures, reminisce on how the concept arose, how difficulties and misconceptions were overcome, how an experimental model was identified, how technologies slowly improved, how experimental evidence gradually built a novel molecular mechanism, how pharmacological modulators were identified, and how these progressed from the lab to the clinic. The concept was unprecedented: a 2.5 million Dalton protease, three tagging enzymes and a large protein tag. However, the most interesting, and unusual aspect of these lectures is the opportunity to see the individual personalities of these investigators. The three interviews bring to the attention of our reader, and to young scientists in general, the determination, perseverance, logic and experimental stringency of young scientists with limited means and unlimited minds. This itself is a unique opportunity for our journal, for which we are extremely grateful and thankful both to The Nobel Foundation, and personally to Drs Hershko, Rose and Ciechanover.

We take this opportunity to stress the involvement of UPS in apoptosis, as previously and elegantly indicated by Alex Varshavsky. ${ }^{8}$ Indeed, UPS is pivotal in regulating degradation of many key regulatory molecules involved in apoptosis. These include p53, p73, mdm2, $1 \mathrm{kB} \alpha, \mathrm{Bax}, \mathrm{Bad}$ and the caspases. In addition, substrates cleaved by caspases undergo a selective degradation thanks to the $\mathrm{N}$-end rule. ${ }^{8}$ This is highlighted by several manuscripts in this issue. ${ }^{9-13}$
However, the most striking example of the involvement of the UPS in apoptosis is provided by the fact that some apoptosisrelated proteins contain domains with E3-like activity owing to a unique C-terminal RING domain, as elegantly described by Vaux, and Silke ${ }^{9}$ and in the Swiss-German humour of Pascal Meier. ${ }^{10}$ These are the inhibitor of apoptosis (IAP) family of proteins, including XIAP, C-IAP-1 and C-IAP-2. Thanks to these special domains, IAPs can regulate the degradation of several proteins, including caspase 9 , which interacts with BIR3, and caspases 3 and 7 , which bind to the flanks of BIR2. CIAP1 and CIAP2 can ubiquitinate TRAF2 and RIP via their BIR2 and BIR3 domains. The activation of the IAP's E3 activity can be triggered by binding with IAP antagonists Smac/Diablo in mammals and Hid, Grim and Reaper in insects - or by IAP's self dimerisation. IAP-binding motifs can be generated by proteolysis, for example, by caspase cleavage; according to Varshavsky, IAP would be acting as E3 N-recognins for a type-3, primary destabilising alanine residue at the amino-termini of proteins with potential IAPbinding motifs. ${ }^{9}$ Inactivation of the proteasome following caspase-mediated cleavage may disable the proteasome, interfering with its role in the regulation of key cellular processes and thereby facilitating an increase in the induction of apoptosis. ${ }^{14}$ This concise look at the $\mathrm{N}$-end rule and the RING E3 activity of IAPs shows how understanding of these functions is just taking its first steps. For example, why does dIAP1 associate with multiple E2s via its RING finger with UBCD1, via UBR1 with UBCD2? And, does dIAP1 also interact with the F-box protein Morgue in the SCF E3 complex? Multiple RING systems (UBR1, SCF E3 complex, dIAP1), and related E2s, are all anchored to dIAP1. Are these three systems isolated or cooperating? And how do they finely tune death wish? ${ }^{15}$

The UPS is involved in several diseases due to its role in protein degradation and misfolding. In particular, Stuart Lipton highlights ubiquitin-related abnormalities in Parkinson's Disease. ${ }^{16}$ UPS is a pivotal mechanism through which cells identify, target, remove and degrade damaged or toxic proteins. This allows tagging of the target molecules with poly-Ub via the E1-E2-E3 system, and their subsequent proteasomal degradation. Very recently, reviewed in Lipton, ${ }^{16}$ oxidative (ROS) and nitroxidative (RNS) insults generated during stress, aging or exposure to environmental insults were found to compromise this defensive mechanism, resulting in accumulation of toxic proteins and eventually leading to pathological conditions, namely protein misfolding diseases such as Parkinson's and Huntington's. Nitric oxide (NO) is a hydrophobic molecule and highly diffusible free radical, generated from the oxidation of L-arginine to L-citrulline by a family of constitutive or cytokine-inducible nictotinamide adenine dinucleotide phosphate (NADPH)-dependent isoenzymes, the NO synthases (NOS). The chemistry of NO 


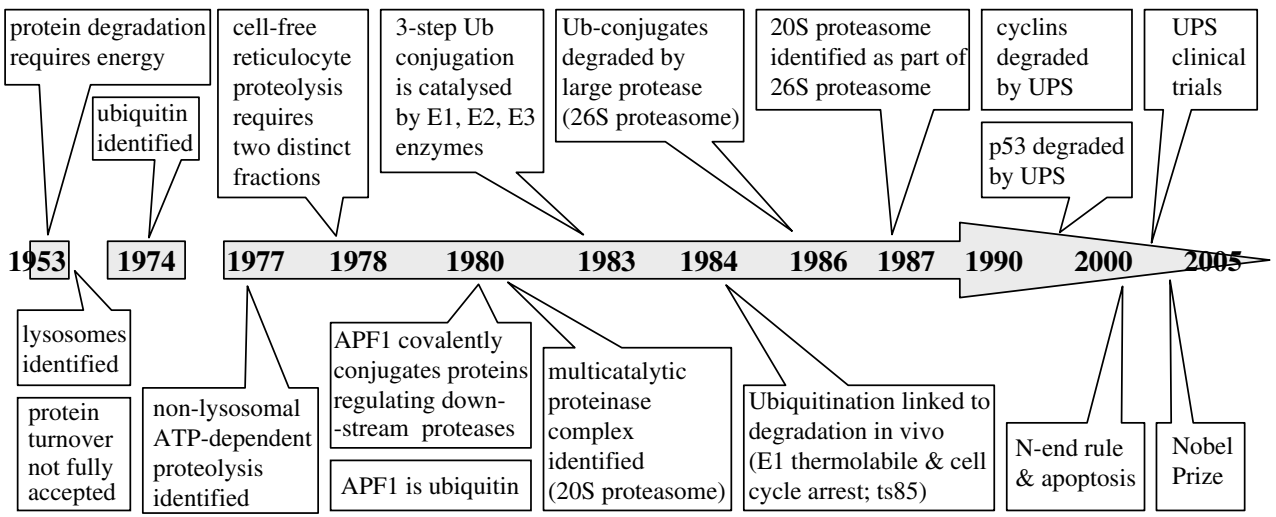

Figure 1 Timeline of the discovery of the UPS. The diagram shows sum of the pivotal discoveries and milestones that historically lead to the identification of the UPS intracellular protein degradation. Owing to obvious space limitation, it is not possible to provide the necessary credit to all scientists and discoveries involved. At present, several diseases related to protein misfolding and defect in protein degradation have been identified, and specific inhibitors of the UPS are now in clinical trials

involves interrelated redox forms $\left(\mathrm{NO} \cdot, \mathrm{NO}^{+}, \mathrm{NO}^{-}\right)$, with different chemical reactivity towards distinct target groups; in particular, S-nitrosylation of cysteine residues is a common protein modification achieved by $\mathrm{NO}$ in the presence of oxygen and often associated with loss of protein function, being also able to regulate apoptosis. ${ }^{17}$ It is therefore not surprising that $\mathrm{NO}$ can S-nitrosylate the Cys' active site of the E3 ubiquitin ligase Parkin, inducing or inactivating the UPS, depending on the timing and magnitude of the insult. ${ }^{16}$ Consequently, its targets, Synphillin-1, Pael-R and CDCrel1, can promote Lewy Bodies-like inclusions (LBs) in cells when coexpressed with $\alpha$-synuclein. The presence of ubiquitinated Parkin substrates in LBs, as well as of S-nitrosylated Parkin and $\mathrm{UCH}-\mathrm{L} 1$ - a deubiquitinating enzyme that recycles ubiquitin - in Parkinson lesions, indicates the clinical relevance of this pathway.

Inhibitors of the proteasome have been developed in the last few years. ${ }^{18}$ Alfred L Goldberg, a pioneer in the field of proteasome research, co-founded MyoGenetics (subsequently absorbed by ProScript and later Millenium) with the original aim of developing proteasome inhibitors to slow the progression of muscle wasting diseases. He recognised that inhibiting the proteasome was an excellent way to study its function. His team identified MG132 (MG standing for MyoGenetics), which remains the most widely used proteasome inhibitor in basic research, and PS-341 (PS standing for ProScript) has been approved for the treatment of multiple myeloma (Phase III clinical trial), and has demonstrated efficacy against other cancers. The original group of enzymologists (led by Ross Stein), chemists (led by Julian Adams) and cell biologists (led by Vito Palombella) at MyoGenetics led to the development of many other UPS inhibitors, such as PS-519, now also in clinical trials. A relevant survival regulator controlled by the UPS is NF- $\kappa \mathrm{B}$, where the inhibitor IkB is ubiquitinated by the $\mathrm{E} 3$ ligase $\beta$ - TrCP - and is thereby targeted for degradation. UPS inhibitors potentiate cisplatin-induced apoptosis, and revert cisplatin drug resistance by inhibiting the NER-dependent repair of cisplatin-DNA adducts via two distinct mechanisms: (i) proteasome-inhibitor-induced depletion of ubiquitinated histone $\mathrm{H} 2 \mathrm{~A}$ in nucleosomes, which promotes chromatin condensation and possibly interferes with the function of DNA damage recognition and repair enzymes; (ii) proteosome inhibitors diminish the excision repair crosscomplementation group 1 (ERCC-1) response to cisplatin, perhaps secondary to changes in chromatin structure that interfere with transcription of the ERCC-1 gene. These inhibitors of the proteasome are therefore powerful inducers of apoptosis. ${ }^{19}$ Henderson et $a l{ }^{19}$ using a novel and elegant methodology show that caspases are involved in cell death induced by proteasome inhibitors, even though the authors have not, strictly speaking, demonstrated their requirement, as blocking caspases delay the apoptotic phenotype, and fail to demonstrate that caspase activation can occur in the absence of apoptosome, for example in caspase 9-/- cells. Despite a marginal effect of Smac/Diablo, the authors demonstrate that proteasome inhibitors can activate caspases, and can induce cell death. The mechanism of death is, however, complex, as ubiquitination plays further roles besides targeting substrates for proteasome-specific degradation, ${ }^{7}$ and Kucharczak ${ }^{12}$ shows that the $\mathrm{Bcl}-2$-related antiapoptotic $\mathrm{Bfl}-1$ undergoes a constitutive ubiquitin/proteasome-mediated turnover which transforms it into a proapoptotic factor. The authors suggest this mechanism to explain antiapoptotic $\mathrm{Bfl}-1$ function induced by stress (staurosporine or cytokine withdrawal) and its proapoptotic effect induced by TNF. Here, however, the physiological role needs further definitive evidence, and the role of calpains requires further refinement. Nonetheless, this novel mechanism could contribute to understanding the already described switch of anti- versus proapoptotic effects of several members of the $\mathrm{Bcl} 2$ family. For these reasons, proteasome inhibitors are now undergoing evaluation in clinical trials focused on several cancers, as elucidated in Salomoni. ${ }^{18}$ Indeed, the regulation of protein stability by the UPS is a critical issue for further understanding the molecular basis of tumorigenesis. Aberrant proteolysis of substrates involved in the regulation of cell cycle, gene transcription and apoptosis has been shown to contribute to carcinogenesis. As a consequence, development of reagents that specifically inhibit components of the ubiquitin system are crucial in analysing molecules of the ubiquitination pathways as potential molecular targets in cancer therapy. As an example, in vitro and in vivo studies have highlighted that proliferating malignant cells are highly sensitive to proteasome blockade- 
mediated killing. In addition, proteasome inhibitor treatment renders cancer cells more responsive to standard chemo- and radiation therapy. A better comprehension of the degradation pathways controlling apoptosis-related protein stability will assist in elucidating the impact of these proteins on tumorigenesis and chemoresistance. Furthermore, understanding how these pathways are regulated in response to DNA damage and how they can be chemically manipulated in order to modulate apoptosis-mediated chemosensitivity may be essential for improving clinical management of tumours currently resistant to therapy.

Five clinical trials based on UPS inhibition are currently in progress: $^{18}$

(i) Velcade-TM (dipeptide boronic acid, Bortezomid, PS341, Millenium Pharmaceuticals Inc.) is in Phase III clinical trials and the FDA has approved it for relapsed and refractory multiple myeloma and other solid cancers. It stabilises cell cycle and proapoptotic proteins, inhibits antiapoptotic proteins and affects tumour microenvironment;

(ii) MLN519 (lactacystin derivative, PS-519, Millenium Pharmaceuticals Inc.) has been approved for a Phase I clinical trial for acute stroke and myocardial infarct; it is a potent anti-inflammatory and neuroprotective compound;

(iii) Epoxomicin-Eponemycin (Streptomyces epoxyketones) is in preclinical development; it has cytotoxic effects in various tumour cells;

(iv) NLVS (trileucine vinyl-sulphone) is also in preclinical development; it is an irreversible inhibitor of trypsin- and chemotrypsin-like proteasome activities;

(v) Ritonavir (Peptidomimetic protease inhibitor; Abbott), a protease inhibitor currently indicated in HIV management, is also in Phase II clinical trials for tumour patients, based on its inhibition of chemotrypsin-like activity in the proteasome.
Only 20 years have passed since the discovery of the UPS by Aaron Ciechanover, Avram Hershko, Irwin Rose, celebrated in this special issue. These new inhibitors represent a new class of anticancer agents, acting on the UPS, undoubtedly providing therapeutically valuable tools. However, to achieve more specificity, and thus increase the safety and efficacy of therapeutic agents targeting the UPS, the intervention of upstream proteasome degradation is desirable, that is ubiquitination by E3 ligase. This suggests, in fact, that possibly the best selectivity should not be searched for at the proteasome level, but rather upstream, at the E3 ligase level. To date no E3-specific inhibitors have been developed. This will be the object of forthcoming research.

We are indebted to The Nobel Foundation for this exceptional opportunity to provide our readers with a unique point of view. We hope our readers will, indeed, perceive the human dimension underpinning these readings and that they will stimulate enthusiasm, perseverance and determination in a new generation of researchers.

1. Ciechanover A (2005) Cell Death Differ. 12: 1167-1177

2. Hershko A (2005) Cell Death Differ. 12: 1158-1161

3. Rose I (2005) Cell Death Differ. 12: 1162-1166

4. Ciechanover A (2005) Cell Death Differ. 12: 1178-1190

5. Hershko A (2005) Cell Death Differ. 12: 1191-1197

6. Rose I (2005) Cell Death Differ. 12: 1198-1201

7. Ciechanover A (2005) Nat. Rev. Mol. Cell Biol. 6: 79-86

8. Varshavsky A (2003) Nat. Cell Biol. 5: 373-376

9. Vaux D and Silke J (2005) Cell Death Differ. 12: 1205-1207

10. Ditzel $M$ and Meier $P$ (2005) Cell Death Differ. 12: 1208-1212

11. Taylor RC et al. (2005) Cell Death Differ. 12: 1213-1217

12. Kucharczak JF et al. (2005) Cell Death Differ. 12: 1225-1239

13. Vandenabeele $P$ et al. (2005) Cell Death Differ. 12: 1219-1224

14. Cohen GM (2005) Cell Death Differ. 12: 1218

15. Melino $G$ (2001) Nature 412: 23

16. Gu Z et al. (2005) Cell Death Differ. 12: 1202-1204

17. Melino $\mathrm{G}$ et al. (1997) Nature 388: 432-433

18. Rossi $\mathrm{M}$ et al. (2005) Cell Death Differ. 12: 1255-1257

19. Henderson CJ et al. (2005) Cell Death Differ. 12: 1240-1254 